\title{
Lack of National Adoption of Evidence-Based Treatment for Resectable Gastric Adenocarcinoma
}

\author{
Tiffany C. Lee ${ }^{1,2} \cdot$ Koffi Wima ${ }^{1,2} \cdot$ Mackenzie C. Morris ${ }^{1,2} \cdot$ Michael E. Johnston $^{1,2} \cdot$ Shimul A. Shah $^{1,2}$. \\ Syed A. Ahmad ${ }^{1,2}$. Sameer H. Patel ${ }^{1,2}$. Gregory C. Wilson ${ }^{1,2}$ (1)
}

Received: 29 May 2020 / Accepted: 6 November 2020 / Published online: 17 November 2020

(C) 2020 The Society for Surgery of the Alimentary Tract

\begin{abstract}
Background Level 1 evidence for multimodal treatment of resectable gastric adenocarcinoma from the Intergroup 0116 (2001) and MAGIC (2006) trials demonstrated survival benefit of adjuvant chemoradiation (CRT) and perioperative chemotherapy, respectively. We evaluated the adoption of evidence-based treatment in the post-MAGIC era and its impact on survival.

Methods A total of 7058 patients with resectable gastric adenocarcinoma undergoing definitive surgical resection between 2004 and 2015 were analyzed using the National Cancer Database.

Results Over the study period, the proportion of patients receiving adjuvant CRT decreased from $19.1 \%$ to $9.1 \%$, while perioperative chemotherapy increased from $1.9 \%$ to $28.6 \%$. Utilization of perioperative chemotherapy surpassed adjuvant CRT in 2011. Evidence-based treatment (either perioperative chemotherapy or adjuvant CRT) had better overall survival (OS) than other treatments for clinical stage II-III patients $(p<0.05)$. On multivariate analysis of the whole study period, evidence-based treatments were associated with better OS (HR $0.67[0.60-0.74], p<0.05)$. Only 360/1262 $(28.5 \%)$ patients in the perioperative chemotherapy group completed postoperative therapy, which was associated with improved OS $(p<0.05)$. For clinical stage III patients $(n=2402)$, only $806(33.6 \%)$ received evidence-based treatment, while $487(22.2 \%)$ underwent surgery alone. On multivariate analysis of these patients between 2010 and 2015, both perioperative chemotherapy (HR 0.49 [0.35-0.68]) and adjuvant CRT (HR $0.31[0.21-0.44]$ ) were associated with better OS than surgery alone $(p<0.05)$.

Conclusions Since the INT-0116 and MAGIC trials, utilization of evidence-based treatments for resectable gastric adenocarcinoma has increased, with perioperative chemotherapy surpassing adjuvant CRT as the preferred practice. However, overall utilization of these regimens remains quite low nationally despite association with improved OS.
\end{abstract}

Keywords Evidence-based practice $\cdot$ Gastric adenocarcinoma $\cdot$ Multimodality therapy $\cdot$ Level 1 evidence

\section{Introduction}

Treatment of resectable gastric adenocarcinoma involves the utilization of multimodality therapies and has evolved over the

Presentation This paper was selected for presentation at The Society for Surgery of the Alimentary Tract 61st Annual Meeting in Chicago, IL, prior to cancellation of the conference due to COVID-19.

Gregory C. Wilson

wilsong3@ucmail.uc.edu

1 Cincinnati Research on Outcomes and Safety in Surgery (CROSS), Cincinnati, OH, USA

2 Department of Surgery, University of Cincinnati College of Medicine, 231 Albert Sabin Way ML 0558, Cincinnati, OH 45267, USA past two decades. ${ }^{1}$ Level 1 evidence for timing of chemotherapy and chemoradiation in relation to surgery comes from two major trials. In 2001, results from the SWOG-directed Intergroup 0116 (INT-0116) trial were published, demonstrating overall survival benefit with the use of adjuvant chemoradiation for patients who had undergone potentially curative resection of their gastric adenocarcinoma. ${ }^{2}$ In 2006, results from the United Kingdom Medical Research Council Adjuvant Gastric Infusional Chemotherapy (MAGIC) trial were published, showing improved survival with perioperative chemotherapy compared with surgery alone for resectable gastric adenocarcinoma. ${ }^{3}$ Current National Comprehensive Cancer Network (NCCN) guidelines incorporate evidence from both of these trials, recommending either of these approaches as appropriate treatment for localized gastric cancer. ${ }^{4}$ 
The time to widespread adoption of level 1 evidence-based practices may be variable and difficult to predict. A study using the Surveillance, Epidemiology, and End Results (SEER) - Medicare linked database from 1991 to 2009 showed an increase in the proportion of patients $>65$ years of age treated with post-operative chemoradiation after publication of the INT-0116 trial, from 13.0\% (between 1991 and 2001) to $25.4 \%$ (between 2006 and 2009). ${ }^{5}$ They also reported a smaller increase in use of preoperative chemotherapy from 1.5\% (between 2002 and 2005) to $4.7 \%$ (between 2006 and 2009), though inclusion of data only up to 2009 may not have allowed sufficient time to allow for widespread changes in practice patterns after the MAGIC trial. Two studies of the American College of Surgeons' National Cancer Database (NCDB) also demonstrated increases in adjuvant therapy after the INT-0116 trial and perioperative chemotherapy after the MAGIC trial. ${ }^{6,7}$ However, none of these studies directly compared outcomes of patients undergoing perioperative chemotherapy versus postoperative chemoradiation, and given the lack of head-to-head trials, the superiority of one approach versus the other remains to be determined. We hypothesized that multidisciplinary treatment for resectable gastric cancer would be widely adopted since the publications of the INT0116 and MAGIC trials and that adherence to these guidelines would result in improved survival for these patients. Therefore, the aims of this study were to (1) describe the changes in treatment practice patterns for resectable gastric adenocarcinomas since publication of the MAGIC trial and (2) to compare survival based on treatment algorithms.

\section{Methods}

\section{Data Source and Patient Selection}

The American College of Surgeons' National Cancer Database (NCDB) is a joint product of the American College of Surgeons Commission on Cancer and the American Cancer Society that includes deidentified patient registry data from more than 1500 nationally accredited cancer programs and captures $70 \%$ of all malignancies diagnosed in the USA. ${ }^{8}$ The NCDB Participant User File was queried for patients with gastric adenocarcinomas who underwent definitive cancer-targeted surgical intervention between 2004 and 2015. Patients were included if they had documented clinical $\mathrm{T}$ stage 2-4 tumors, with any $\mathrm{N}$ stage, but no distant metastases (M0). Patients with missing clinical staging data or under the age of 18 years were excluded. Additionally, patients who had partial esophageal resection as part of their definitive cancer-targeted surgical intervention were also excluded in order to maintain a homogenous cohort, as these patients likely represent gastroesophageal junction tumors which represent a different tumor biology and are more likely to be treated as esophageal cancer. Institutional Review Board exemption status was obtained prior to initiation of the study.

\section{Data Variables}

The following patient characteristics were examined: age (years), gender, race (white, black, Asian, or other/unknown), Charlson-Deyo Comorbidity Score (CDCC), income, and insurance status. Additionally, the following tumor features were examined: date of diagnosis, clinical stage, pathologic stage, tumor site (cardia/fundus, body/antrum, unknown), grade (well, moderately, or poorly differentiated), and tumor size $(<2 \mathrm{~cm}, 2-5 \mathrm{~cm}$, or $>5 \mathrm{~cm})$. Surgical details included facility type, location (urban vs. rural), type of resection (total/ near total gastrectomy or partial gastrectomy), number of regional lymph nodes retrieved, regional lymph node status (positive or negative), and margin status (R0 for no residual tumor, R1 for microscopic residual tumor, or R2 for macroscopic residual tumor). The utilization of chemotherapy and radiation therapy was each categorized into neoadjuvant, adjuvant, or none. Evidence-based treatment was defined as either perioperative chemotherapy (neoadjuvant chemotherapy was used as a surrogate for perioperative chemotherapy, with the assumption that those receiving neoadjuvant chemotherapy were intended to also receive adjuvant chemotherapy) or adjuvant chemoradiation. Neoadjuvant chemoradiation was considered as a separate category as this regimen, although included in the NCCN guidelines as a category 2B recommendation due to lack of level 1 evidence supporting its use, has been advocated for use at several centers and based on available literature is more likely to be used for proximal tumors. All other treatment regimens were grouped into an "other" treatment cohort. Outcomes including 30-day and 90-day mortality were examined. The primary outcome of overall survival was calculated using last contact or death date.

\section{Statistical Analysis}

Statistical analyses were performed via statistical programs SAS 9.4 (SAS Institute, Cary, NC, USA). Data collected were grouped into continuous and categorical variables as appropriate. Continuous variables were described as estimates of central tendency (median) and interquartile range (IQR). Categorical variables were described as integers and percentages (\%). Categorical variables were analyzed using Pearson's Chi-squared test or Fisher's exact test when appropriate, while continuous variables were compared through Wilcoxon ranksum test. Characteristics were compared between patients diagnosed between 2004 and 2009 versus 2010 and 2015 using univariate analyses. Similar univariate analyses were used to compare patients who underwent evidence-based treatment 
Fig. 1 Consort diagram of patient selection criteria

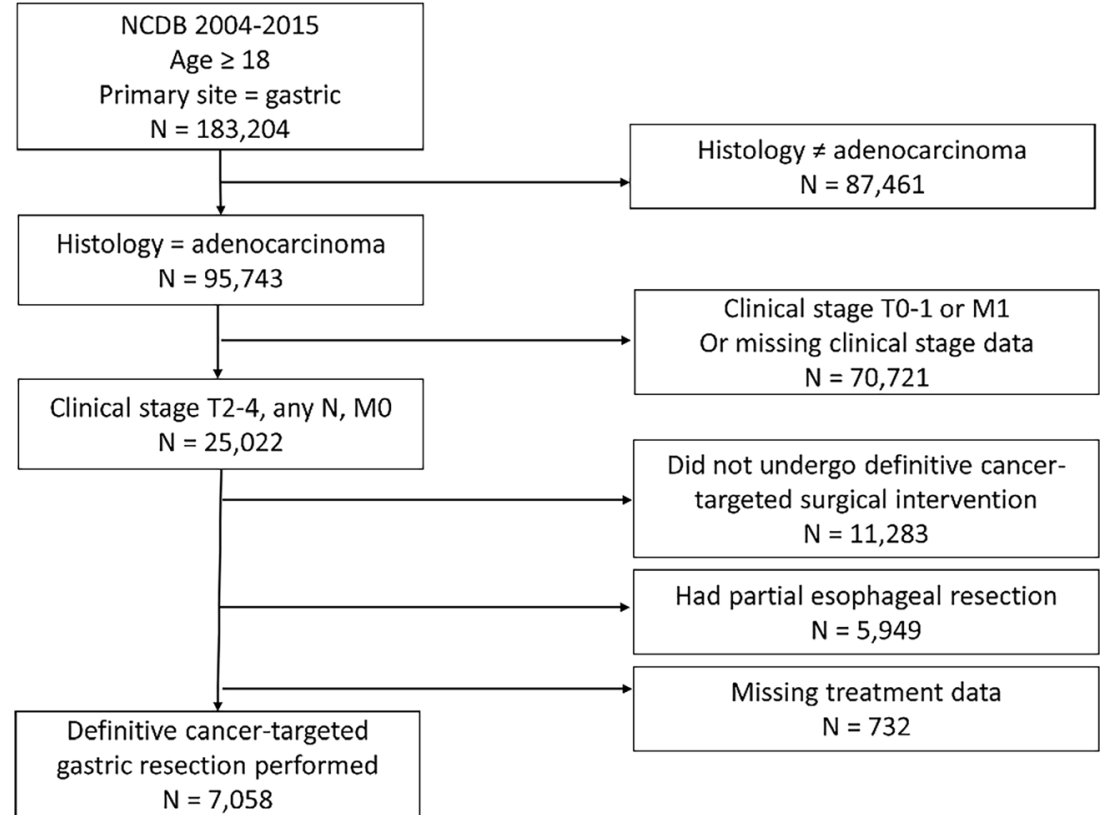

versus neoadjuvant chemoradiation versus other treatments. Multivariate Cox regression analysis for predictors of overall survival was performed, including time period (2004-2009 vs. 2010-2015), ethnicity, income, insurance, facility type, location, pathologic stage, tumor site, grade, treatment regimen, type of resection, lymph node status, and margin status. A separate Cox regression analysis was performed for overall survival in clinical stage III patients between 2010 and 2015 to evaluate impact after both the INT-0116 and MAGIC trials. Kaplan-Meier analyses were performed comparing overall and stage-specific survival based on treatment regimen status. Variables with a $p$ value $<0.05$ were determined to be statistically significant.

\section{Results}

\section{Entire Cohort Analyses}

A total of 7058 patients met inclusion criteria and were included in the analysis (Fig. 1). Over the study time period, the proportion of patients receiving evidence-based treatment (either perioperative chemotherapy or adjuvant chemoradiation) increased from 21.0 to $37.7 \%$, while neoadjuvant chemoradiation also increased from 17.5 to $34.0 \%$, and other treatments decreased from 61.5 to $28.4 \%$ (Fig. 2). The use of surgery alone decreased over the study time period; however, it remained the treatment for $25.5 \%$ of patients (down from
Fig. 2 Trends in treatment patterns over time, comparing evidence-based regimens (perioperative chemotherapy or adjuvant chemoradiation) vs. neoadjuvant chemoradiation vs. surgery alone vs other treatments

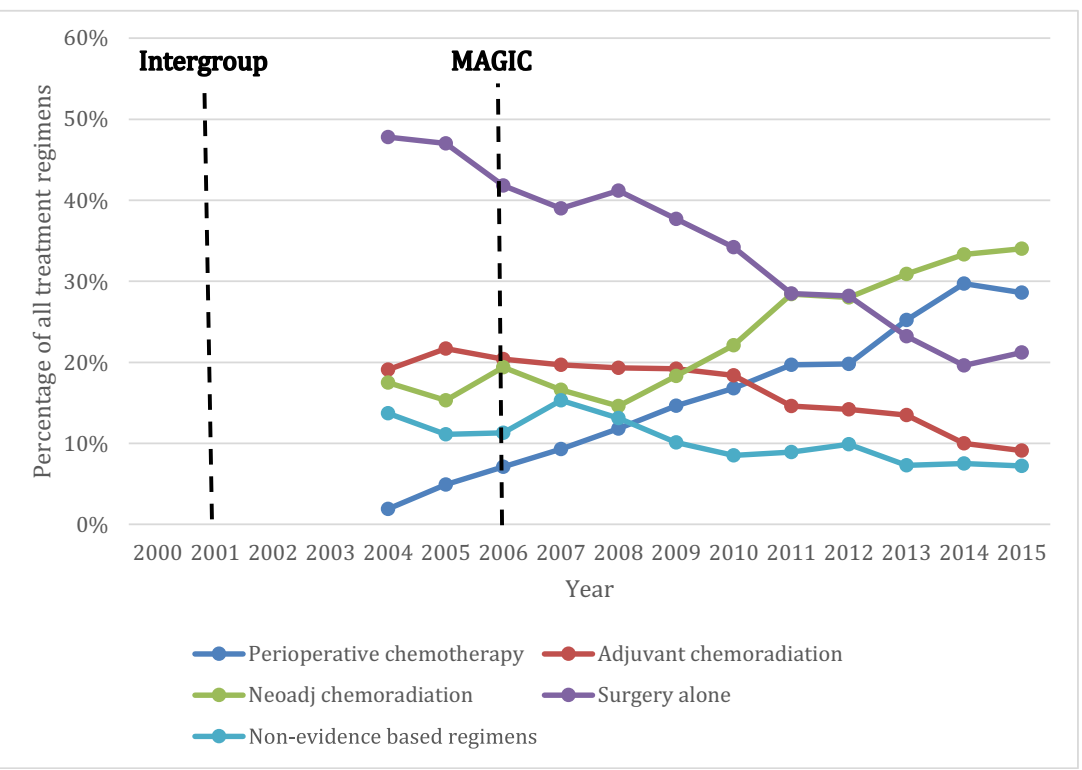


Table 1 Comparison of patient and tumor characteristics between the cohorts diagnosed between 2004 and 2009 vs. 2010 and 2015

\begin{tabular}{|c|c|c|c|c|c|c|c|}
\hline \multicolumn{4}{|l|}{ cohorts diagnosed b } & & \multirow[b]{2}{*}{$\begin{array}{l}2004-2009 \\
(n=2821) N(\%) \\
\text { or median (IQR) }\end{array}$} & \multirow[b]{2}{*}{$\begin{array}{l}2010-2015 \\
(n=4237) \\
N(\%) \text { or } \\
\text { median (IQR) }\end{array}$} & \multirow[b]{2}{*}{$p$ value } \\
\hline & $\begin{array}{l}2004-2009 \\
(n=2821) N(\%) \\
\text { or median (IQR) }\end{array}$ & $\begin{array}{l}2010-2015 \\
(n=4237) \\
N(\%) \text { or } \\
\text { median (IOR) }\end{array}$ & $p$ value & & & & \\
\hline Age & $68(59-77)$ & $67(58-75)$ & $<0.01$ & $\begin{array}{l}\text { Adjuvant } \\
\text { chemotherapy }\end{array}$ & $246(8.7 \%)$ & $303(7.2 \%)$ & \\
\hline Male gender & $1872(66.4 \%)$ & $2995(70.7 \%)$ & $<0.01$ & Adjuvant radiation & $101(3.6 \%)$ & $43(1.0 \%)$ & \\
\hline Ethnicity & & & 0.16 & Tumor site & & & $<0.01$ \\
\hline Caucasian & $2149(76.9 \%)$ & $3281(78.0 \%)$ & & Body/antrum & $729(25.8 \%)$ & $1086(25.6 \%)$ & \\
\hline Black & $421(15.1 \%)$ & $579(13.8 \%)$ & & Cardia/fundus & $1013(35.9 \%)$ & $1928(45.5 \%)$ & \\
\hline Asian & $185(6.6 \%)$ & $260(6.2 \%)$ & & Other/unknown & $1079(38.3 \%)$ & $1223(28.9 \%)$ & \\
\hline American Indian & $7(0.3 \%)$ & $13(0.3 \%)$ & & Grade & & & $<0.01$ \\
\hline Other/unknown & $32(1.2 \%)$ & $72(1.7 \%)$ & & Well-differentiated & $116(4.4 \%)$ & $172(4.4 \%)$ & \\
\hline Insurance & & & 0.07 & $\begin{array}{l}\text { Moderately } \\
\text { differentiated }\end{array}$ & $821(30.8 \%)$ & $1409(35.9 \%)$ & \\
\hline Private & $975(35.6 \%)$ & $1527(36.4 \%)$ & & Poorly differentiated & $1684(63.2 \%)$ & $2310(58.8 \%)$ & \\
\hline Medicaid & $155(5.7 \%)$ & $290(6.9 \%)$ & & Unknown & $45(1.7 \%)$ & $36(0.9 \%)$ & \\
\hline Medicare & $1503(54.9 \%)$ & $2193(52.3 \%)$ & & Type of resection & & & 0.36 \\
\hline $\begin{array}{l}\text { Not insured } \\
\text { Unknown }\end{array}$ & $\begin{array}{l}79(2.9 \%) \\
27(1.0 \%)\end{array}$ & $\begin{array}{l}129(3.1 \%) \\
57(1.4 \%)\end{array}$ & & $\begin{array}{l}\text { Total/near total } \\
\text { gastrectomy }\end{array}$ & $705(26.8 \%)$ & $1029(25.8 \%)$ & \\
\hline Income & & & 0.54 & Partial gastrectomy & $1928(73.2 \%)$ & $2966(74.2 \%)$ & \\
\hline$<\$ 30,000$ & $411(15.2 \%)$ & $589(14.4 \%)$ & & Lymph nodes & & & $<0.01$ \\
\hline$\$ 30,000-34,999$ & $475(17.6 \%)$ & $717(17.5 \%)$ & & ${ }_{0}^{\text {examined }}$ & $265(94 \%)$ & $280(66 \%)$ & \\
\hline$\$ 35,000-\$ 45,999$ & $725(26.9 \%)$ & $1160(28.4 \%)$ & & $1-14$ & $1048(37.2 \%)$ & $1242(29.3 \%)$ & \\
\hline$\$ 46,000+$ & $1085(40.2 \%)$ & $1624(39.7 \%)$ & & $15+$ & $1449(51.4 \%)$ & $2651(62.6 \%)$ & \\
\hline Facility type & $244(98 \%)$ & $311(83 \%)$ & $<0.01$ & Unknown & $59(2.1 \%)$ & $64(1.5 \%)$ & \\
\hline $\begin{array}{l}\text { Community } \\
\text { cancer programs }\end{array}$ & $244(9.8 \%)$ & & & $\begin{array}{l}\text { Positive lymph } \\
\text { nodes }\end{array}$ & $1643(64.6 \%)$ & $2113(53.7 \%)$ & $<0.01$ \\
\hline $\begin{array}{l}\text { Academic/research } \\
\text { program }\end{array}$ & $1150(46.2 \%)$ & $1918(51.3 \%)$ & & Margin status & & & $<0.01$ \\
\hline Integrated Network & $1094(44.0 \%)$ & $1508(40.4 \%)$ & & R0 & $2308(90.8 \%)$ & $3670(93.6 \%)$ & \\
\hline Cancer Program & & & & $\mathrm{R} 1$ & $193(7.6 \%)$ & $227(5.8 \%)$ & \\
\hline Location & & & 0.35 & $\mathrm{R} 2$ & $40(1.6 \%)$ & $26(0.7 \%)$ & \\
\hline
\end{tabular}

Rural

Charlson-Deyo Score

1

2

3

Clinical stage

I

II

III

IV

Treatment regimen

Surgery alone

Perioperative chemotherapy

Adjuvant chemoradiation

Neoadjuvant chemoradiation

Table 1 (continued)
$1985(70.4 \%) \quad 2890(68.2 \%)$

$620(22.0 \%) \quad 992(23.4 \%)$

$167(5.9 \%) \quad 261(6.2 \%)$

$49(1.7 \%) \quad 94(2.2 \%)$

$704(26.2 \%) \quad 661(15.8 \%)$

$832(31.0 \%) \quad 2024(48.4 \%)$

$924(34.4 \%) \quad 1478(35.4 \%)$

$226(8.4 \%) \quad 16(0.4 \%)$

$1175(41.7 \%) \quad 1080(25.5 \%)$

$264(9.4 \%) \quad 998(23.6 \%)$

$559(19.8 \%) \quad 556(13.1 \%)$

$476(16.9 \%) \quad 1257(29.7 \%)$
$41.7 \%$ of patients in the early time period). For the evidencebased treatment regimens specifically, the proportion of patients receiving perioperative chemotherapy increased from 1.9 to $28.6 \%$ over the study period, while those receiving adjuvant chemoradiation decreased from 19.1 to $9.1 \%$. Characteristics of patients in this study broken down by time period are listed in Table 1. Overall and stage-specific (clinical stage I-III) survival was better in the latter time period on Kaplan-Meier analysis (Fig. 3).

Patient and tumor characteristics broken down by treatment regimen are listed in Table 2 . The neoadjuvant chemoradiation cohort consisted mostly of cardia/fundus tumors which was different than the other two cohorts which tended to be more equally distributed. Thirty- and 90-day post-surgical mortality was higher in the non-evidence-based group $(7.7 \%$ and $15.2 \%$ ) compared with the neoadjuvant chemoradiation (3.6\% and $7.0 \%$ ) or evidence-based regimen (1.2\% and 3.1\%) groups $(p<0.01)$. Kaplan-Meier survival curves by treatment 


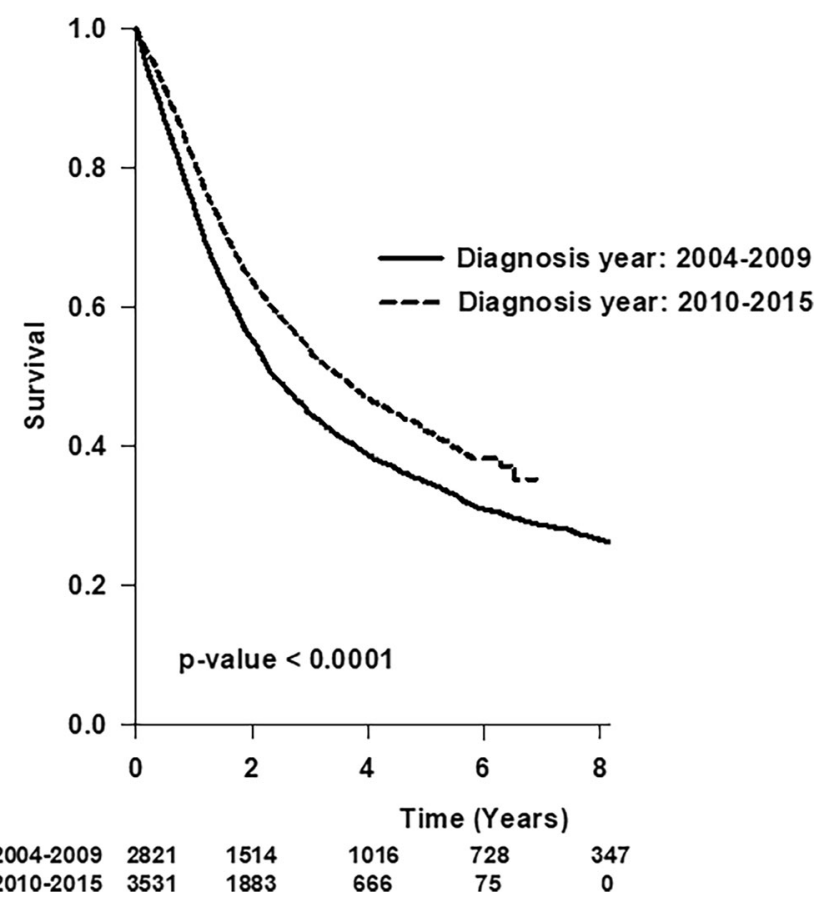

Fig. 3 Comparison of overall survival along the study timeline. KaplanMeier survival curves for patients diagnosed between 2004 and 2009 versus 2010 and 2015, including all clinical stages. Comparison between groups performed using log rank analysis

regimen are shown in Fig. 4, with improved overall survival in the evidence-based cohort (Fig. 4). The most notable differences were seen in the clinical stage III patients (not shown).

A multivariate Cox regression analysis for factors associated with overall survival in the whole cohort was performed (Table 3). Factors associated with worse overall survival included older age, higher CDCC scores, higher pathologic stage, poorly differentiated tumors, positive lymph nodes, and positive margin status. Both the neoadjuvant chemoradiation and other regimen cohorts were associated with worse survival compared with the evidence-based regimen cohort.

\section{Subgroup Analyses: Clinical Stage III Patients Diagnosed Between 2010 and 2015}

The most notable survival differences were seen in the clinical stage III patients; therefore, we performed a subgroup analysis comparing the INT-0116 versus MAGIC regimens for these patients in the modern cohort. Only $33.6 \%(n=806)$ of all clinical stage III patients received evidence-based treatment, while $22.2 \%(n=487)$ underwent surgery alone. Patient and tumor characteristics for this subgroup analysis are listed in Table 4. Patients in the perioperative chemotherapy cohort were more likely to be Caucasian, treated at an academic facility, undergo total/near total gastrectomy, have an $\mathrm{R} 0$ resection, and lower proportion of positive lymph nodes. Perioperative chemotherapy was associated with improved overall survival compared with the adjuvant chemoradiation cohort (Fig. 5). On Cox regression analysis, factors associated with better overall survival included any multimodal treatment regimen other than surgery alone, including perioperative chemotherapy (HR 0.49 [0.35-0.68], $p<0.01$ ), adjuvant chemoradiation (HR 0.31 [0.21-0.44], $p<0.01$ ), and neoadjuvant chemoradiation (HR $0.55[0.40-0.76], p<0.01)($ Table 5)

\section{Perioperative Chemotherapy Cohort}

Of note, out of 1262 patients in the perioperative chemotherapy cohort across the entire time period, only 360 (28.5\%) completed the regimen and received adjuvant chemotherapy. Completion of perioperative chemotherapy was associated with improved overall survival compared with patient who did not complete the therapy (median overall survival 7.09 [4.68-not reached] years vs. $4.36[3.56-5.48]$ years, $p=$ 0.002 ). Additionally, the effect of down-staging on survival was examined. A total of $58.4 \%$ of patients in the perioperative chemotherapy cohort were down-staged after preoperative chemotherapy. Of the stage three patients, $32.7 \%$ were down-staged to $2,15.9 \%$ were down-staged to 1 , and $51.4 \%$ remained stage 3. Down-staging after preoperative chemotherapy was associated with better survival than those who were not down-staged (Fig. 6), with 5-year survival of $65.1 \%$ in the down-staged group versus $37.0 \%$ in patients not down-staged.

\section{Discussion}

In this study, we demonstrated an increase in utilization of evidence-based multimodal therapy for the treatment of resectable gastric adenocarcinoma using the NCDB. Since publication of the MAGIC trial results, use of perioperative chemotherapy has overtaken adjuvant chemoradiation as the preferred treatment regimen. This trend will likely continue based on recent results from the FLOT4 study which further solidified the beneficial role of perioperative chemotherapy in the management of resectable, locally advanced gastric adenocarcinoma. ${ }^{9}$ Use of either of these regimens was associated with better overall survival compared with other treatments in the whole study cohort as well as specifically for patients with clinical stage III tumors. Despite this, still over one quarter of all patients underwent surgery alone in the modern time period. However, among patients in the perioperative chemotherapy cohort, only $28.5 \%$ completed adjuvant chemotherapy. Receipt of both neoadjuvant and adjuvant chemotherapy was associated with improved overall survival compared with that only receiving neoadjuvant chemotherapy. Not surprisingly, down-staging after neoadjuvant chemotherapy was also associated with better survival.

There is a wide range in the time lag between publication of level 1 evidence to widespread adoption of evidence-based 
Table 2 Patient and tumor characteristics by treatment regimen including evidence-based regimens (perioperative chemotherapy or adjuvant chemoradiation) versus neoadjuvant chemoradiation versus non-evidence-based (adjuvant chemotherapy, adjuvant radiation, or surgery alone) regimens

Evidence-based (perioperative chemotherapy or adjuvant chemoradiation) $(n=2377)$ $N(\%)$ or median (IQR)
Neoadjuvant chemoradiation Non-evidence based treatment $p$ value $(n=1733) N(\%)$ or median $\quad(n=2948) N(\%)$ or median (IQR) (IQR)

\begin{tabular}{|c|c|c|c|c|}
\hline \multicolumn{4}{|l|}{ Time period } & \multirow[t]{3}{*}{$<0.01$} \\
\hline 2004-2009 & $823(34.6 \%)$ & $476(27.5 \%)$ & $1522(51.6 \%)$ & \\
\hline 2010-2015 & $1554(65.4 \%)$ & $1257(72.5 \%)$ & $1426(48.4 \%)$ & \\
\hline Age & $65(56-72)$ & $63(56-70)$ & $73(64-81)$ & $<0.01$ \\
\hline Male gender & $1629(68.5 \%)$ & $1457(84.1 \%)$ & $1781(60.4 \%)$ & $<0.01$ \\
\hline Ethnicity & & & & $<0.01$ \\
\hline Caucasian & $1681(71.3 \%)$ & $1591(92.5 \%)$ & $2158(73.9 \%)$ & \\
\hline Black & $420(17.8 \%)$ & $90(5.2 \%)$ & $490(16.8 \%)$ & \\
\hline Asian & $199(8.4 \%)$ & $27(1.6 \%)$ & $219(7.5 \%)$ & \\
\hline American Indian & $10(0.4 \%)$ & $3(0.2 \%)$ & $7(0.2 \%)$ & \\
\hline Other/unknown & $48(2.0 \%)$ & $9(0.5 \%)$ & $47(1.6 \%)$ & \\
\hline Insurance & & & & $<0.01$ \\
\hline Private & $981(42.1 \%)$ & $853(49.7 \%)$ & $668(23.2 \%)$ & \\
\hline Medicaid & $180(7.7 \%)$ & $89(5.2 \%)$ & $176(6.1 \%)$ & \\
\hline Medicare & $1051(45.1 \%)$ & $710(41.4 \%)$ & $1935(67.1 \%)$ & \\
\hline Not insured & $97(4.2 \%)$ & $30(1.8 \%)$ & $81(2.8 \%)$ & \\
\hline Unknown & $24(1.0 \%)$ & $35(2.0 \%)$ & $25(0.9 \%)$ & \\
\hline Income & & & & $<0.01$ \\
\hline$<\$ 30,000$ & $322(14.1 \%)$ & $177(10.6 \%)$ & $501(17.7 \%)$ & \\
\hline$\$ 30,000-34,999$ & $386(16.9 \%)$ & $298(17.8 \%)$ & $508(18.0 \%)$ & \\
\hline$\$ 35,000-\$ 45,999$ & $631(27.6 \%)$ & $492(29.5 \%)$ & $762(27.0 \%)$ & \\
\hline$\$ 46,000+$ & $950(41.5 \%)$ & $703(42.1 \%)$ & $1056(37.4 \%)$ & \\
\hline Facility type & & & & $<0.01$ \\
\hline Academic/research program & $1089(52.2 \%)$ & $855(57.0 \%)$ & $1124(42.6 \%)$ & \\
\hline Community cancer programs & $169(8.1 \%)$ & $101(6.7 \%)$ & $285(10.8 \%)$ & \\
\hline Integrated Network Cancer Program & $827(39.7 \%)$ & $543(36.2 \%)$ & $1232(46.7 \%)$ & \\
\hline Location & & & & 0.17 \\
\hline Rural & $31(1.4 \%)$ & $30(1.8 \%)$ & $58(2.0 \%)$ & \\
\hline Urban & $2273(98.7 \%)$ & $1648(98.2 \%)$ & $2789(98.0 \%)$ & \\
\hline Charlson-Deyo Comorbidity Score & & & & $<0.01$ \\
\hline 0 & $1713(72.1 \%)$ & $1259(72.7 \%)$ & $1903(64.6 \%)$ & \\
\hline 1 & $524(22.0 \%)$ & $365(21.1 \%)$ & $723(24.5 \%)$ & \\
\hline 2 & $109(4.6 \%)$ & $80(4.6 \%)$ & $239(8.1 \%)$ & \\
\hline 3 & $31(1.3 \%)$ & $29(1.7 \%)$ & $83(2.8 \%)$ & \\
\hline Clinical stage & & & & $<0.01$ \\
\hline I & $347(14.9 \%)$ & $134(7.9 \%)$ & $884(31.2 \%)$ & \\
\hline II & $1098(47.2 \%)$ & $670(39.4 \%)$ & $1088(38.4 \%)$ & \\
\hline III & $806(34.6 \%)$ & $871(51.2 \%)$ & $725(25.6 \%)$ & \\
\hline IV & $76(3.3 \%)$ & $26(1.5 \%)$ & $140(4.9 \%)$ & \\
\hline Tumor site & & & & $<0.01$ \\
\hline Body/antrum & $762(32.1 \%)$ & $53(3.1 \%)$ & $1000(33.9 \%)$ & \\
\hline Cardia/fundus & $642(27.0 \%)$ & $1590(91.8 \%)$ & $709(24.1 \%)$ & \\
\hline Unknown & $973(40.9 \%)$ & $90(5.2 \%)$ & $1239(42.0 \%)$ & \\
\hline Grade & & & & $<0.01$ \\
\hline Well-differentiated & $66(3.0 \%)$ & $82(5.4 \%)$ & $140(4.9 \%)$ & \\
\hline
\end{tabular}


Table 2 (continued)

\begin{tabular}{|c|c|c|c|c|}
\hline & $\begin{array}{l}\text { Evidence-based (perioperative } \\
\text { chemotherapy or } \\
\text { adjuvant chemoradiation) }(n=2377) \\
N(\%) \text { or median (IQR) }\end{array}$ & $\begin{array}{l}\text { Neoadjuvant chemoradiation } \\
(n=1733) N(\%) \text { or median } \\
\text { (IQR) }\end{array}$ & $\begin{array}{l}\text { Non-evidence based treatment } \\
(n=2948) N(\%) \text { or median } \\
(\mathrm{IQR})\end{array}$ & $p$ value \\
\hline Moderately differentiated & $662(29.8 \%)$ & $624(41.2 \%)$ & $944(33.1 \%)$ & \\
\hline Poorly differentiated & $1467(66.0 \%)$ & $796(52.6 \%)$ & $1731(60.6 \%)$ & \\
\hline Unknown & $29(1.3 \%)$ & $12(0.8 \%)$ & $40(1.4 \%)$ & \\
\hline Type of resection & & & & $<0.01$ \\
\hline Total/near total gastrectomy & $755(33.4 \%)$ & $338(21.1 \%)$ & $641(23.2 \%)$ & \\
\hline Partial gastrectomy & $1507(66.6 \%)$ & $1268(79.0 \%)$ & $2119(76.8 \%)$ & \\
\hline Lymph nodes examined & & & & $<0.01$ \\
\hline 0 & $116(4.9 \%)$ & $159(9.2 \%)$ & $270(9.2 \%)$ & \\
\hline $1-14$ & $605(25.5 \%)$ & $623(36.0 \%)$ & $1062(36.0 \%)$ & \\
\hline $15+$ & $1625(68.4 \%)$ & $893(51.5 \%)$ & $1582(53.7 \%)$ & \\
\hline Unknown & $31(1.3 \%)$ & $58(3.4 \%)$ & $34(1.2 \%)$ & \\
\hline Positive lymph nodes & $1476(65.6 \%)$ & $696(44.7 \%)$ & $1584(59.3 \%)$ & $<0.01$ \\
\hline Margin status & & & & $<0.01$ \\
\hline R0 & $2009(92.2 \%)$ & $1578(96.5 \%)$ & $2391(90.3 \%)$ & \\
\hline $\mathrm{R} 1$ & $155(7.1 \%)$ & $54(3.3 \%)$ & $211(8.0 \%)$ & \\
\hline $\mathrm{R} 2$ & $15(0.7 \%)$ & $4(0.2 \%)$ & $47(1.8 \%)$ & \\
\hline
\end{tabular}

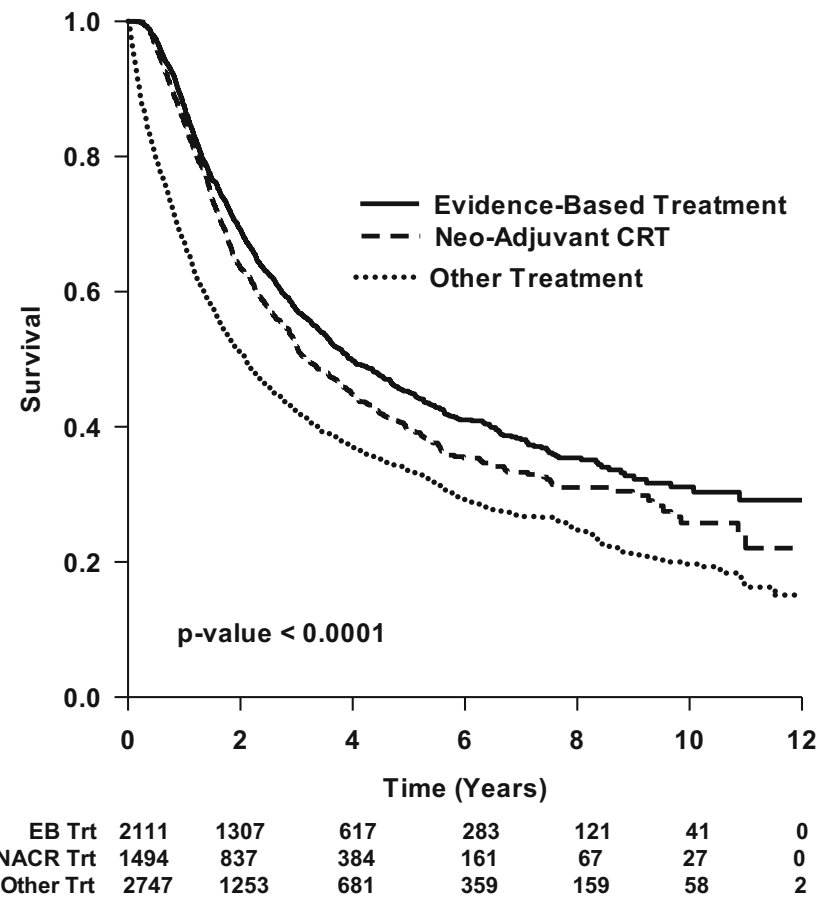

Fig. 4 Survival comparison by treatment strategy. Kaplan-Meier curves demonstrating overall survival of patients who underwent evidence-based treatment (perioperative chemotherapy or adjuvant chemoradiation) versus neoadjuvant chemoradiation versus other treatment regimens, for all clinical stages. $P$ values reflect comparison across all three groups. There was also a significant difference $(p<0.05)$ between the evidence-based treatment and neoadjuvant chemoradiation groups practice, with some literature suggesting an average of 17 years. ${ }^{10}$ In this study, we found that adoption of the MAGIC perioperative chemotherapy regimen surpassed the INT-0116 adjuvant chemoradiation regimen in 2011, 5 years after publication of the MAGIC trial. Unfortunately, there is a lack of head to head trials comparing these regimens, and therefore, we must draw conclusions using retrospective cohort data. ${ }^{11}$ However, the NCDB is a large national database which provides a reasonable view of real-world practice patterns. Previous studies using earlier NCDB cohorts have shown mixed results when comparing the two regimens. One study demonstrated a survival advantage for perioperative chemotherapy, especially in patients who were down-staged from lymph node-positive to lymph node-negative disease after neoadjuvant chemotherapy. ${ }^{12}$ Another study found an improved overall survival with adjuvant chemoradiation, particularly in patients with margin positive resections. ${ }^{13}$ Another study using a California cancer registry found similar outcomes between perioperative chemotherapy and adjuvant chemoradiation for clinically node-positive patients, though chemoradiation resulted in better survival for node-negative patients or those with signet ring cell histology. ${ }^{14}$ Our current study provides an updated analysis with a more contemporary cohort, demonstrating an association between use of evidence-based treatment regimens and overall survival. Furthermore, use of perioperative chemotherapy was associated with better overall survival than adjuvant chemoradiation. 
Table 3 Multivariate Cox regression analysis for predictors of overall survival in entire patient cohort

\begin{tabular}{|c|c|c|}
\hline & HR $(95 \% \mathrm{CI})$ & $p$ value \\
\hline \multicolumn{3}{|l|}{ Time period } \\
\hline 2004-2009 & Ref. & \\
\hline $2010-2015$ & $0.92(0.83-1.01)$ & 0.09 \\
\hline Age & $1.02(1.01-1.02)$ & $<0.01$ \\
\hline \multicolumn{3}{|l|}{ Gender } \\
\hline Male & Ref. & \\
\hline Female & $0.94(0.85-1.04)$ & 0.22 \\
\hline \multicolumn{3}{|l|}{ Ethnicity } \\
\hline Caucasian & Ref. & \\
\hline Black & $0.97(0.84-1.11)$ & 0.68 \\
\hline Asian & $0.73(0.60-0.87)$ & $<0.01$ \\
\hline American Indian & $3.68(1.45-7.61)$ & $<0.01$ \\
\hline Other/unknown & $0.55(0.34-0.84)$ & $<0.01$ \\
\hline \multicolumn{3}{|l|}{ Insurance } \\
\hline Private & Ref. & \\
\hline Medicaid & $0.95(0.76-1.16)$ & 0.60 \\
\hline Medicare & $1.10(0.97-1.23)$ & 0.14 \\
\hline Not insured & $1.21(0.90-1.58)$ & 0.19 \\
\hline Unknown & $1.07(0.65-1.66)$ & 0.76 \\
\hline \multicolumn{3}{|l|}{ Income } \\
\hline$<\$ 30,000$ & Ref. & \\
\hline$\$ 30,000-34,999$ & $0.97(0.83-1.13)$ & 0.67 \\
\hline$\$ 35,000-\$ 45,999$ & $0.89(0.77-1.03)$ & 0.11 \\
\hline$\$ 46,000+$ & $0.90(0.78-1.03)$ & 0.12 \\
\hline \multicolumn{3}{|l|}{ Facility type } \\
\hline Academic/research program & Ref. & \\
\hline Community cancer programs & $1.13(0.96-1.31)$ & 0.14 \\
\hline Integrated Network Cancer Program & $1.11(1.01-1.21)$ & $<0.01$ \\
\hline \multicolumn{3}{|l|}{ Charlson-Deyo Score } \\
\hline 0 & Ref. & \\
\hline 1 & $1.06(0.95-1.18)$ & 0.31 \\
\hline 2 & $1.33(1.11-1.57)$ & $<0.01$ \\
\hline 3 & $1.84(1.35-2.44)$ & $<0.01$ \\
\hline \multicolumn{3}{|l|}{ Pathologic stage } \\
\hline 0 & $1.17(0.66-1.91)$ & 0.55 \\
\hline I & Ref. & \\
\hline II & $1.41(1.20-1.66)$ & $<0.01$ \\
\hline III & $2.46(2.05-2.96)$ & $<0.01$ \\
\hline IV & $3.67(2.94-4.57)$ & $<0.01$ \\
\hline \multicolumn{3}{|l|}{ Treatment regimen } \\
\hline $\begin{array}{l}\text { Evidence-based (perioperative } \\
\text { chemotherapy or adjuvant } \\
\text { chemoradiation) }\end{array}$ & Ref. & \\
\hline Neoadjuvant chemoradiation & $1.50(1.29-1.75)$ & $<0.01$ \\
\hline Other & $1.50(1.35-1.67)$ & $<0.01$ \\
\hline \multicolumn{3}{|l|}{ Tumor site } \\
\hline Body/antrum & Ref. & \\
\hline Cardia/fundus & $1.16(1.01-1.32)$ & 0.03 \\
\hline
\end{tabular}

Table 3 (continued)

\begin{tabular}{llc}
\hline & HR $(95 \%$ CI $)$ & $p$ value \\
\hline Other/unknown & $1.05(0.94-1.18)$ & 0.41 \\
Grade & & \\
Well-differentiated & Ref. & \\
Moderately differentiated & $1.14(0.88-1.51)$ & 0.34 \\
Poorly differentiated & $1.39(1.08-1.84)$ & 0.01 \\
Unknown & $1.28(0.82-1.98)$ & 0.27 \\
Type of resection & & \\
Total/near total gastrectomy & Ref. & \\
Partial gastrectomy & $0.85(0.77-0.94)$ & $<0.01$ \\
Lymph nodes examined & & \\
1-14 & Ref. & $<0.01$ \\
15+ & $0.75(0.68-0.82)$ & $<0.01$ \\
Positive lymph nodes & $1.33(1.17-1.53)$ & \\
Margin status & & $<0.01$ \\
R0 & Ref. & $<0.01$ \\
R1 & $1.52(1.30-1.77)$ & \\
R2 & $1.98(1.32-2.85)$ & \\
\hline
\end{tabular}

Other trials have attempted to compare chemotherapy and chemoradiation more specifically in the adjuvant setting. The Adjuvant Chemoradiotherapy in Stomach Tumors (ARTIST) trial, published in 2015, comparing adjuvant chemotherapy versus chemoradiation following D2-resected gastric cancer found similar overall survival between the two treatments. ${ }^{15}$ While not powered to specifically address this issue, there did appear to be improved disease-free survival in subgroup analysis of patients with positive lymph nodes. Interim analysis of the follow-up ARTIST 2 trial focusing on node-positive tumors also showed no difference in disease-free survival between adjuvant chemotherapy and chemoradiation groups. ${ }^{16}$ The CRITICS trial, published in 2018, compared adjuvant chemotherapy versus chemoradiation after neoadjuvant chemotherapy and resection, with results showing no difference in overall survival. ${ }^{17}$ Of note, only $59 \%$ of the chemotherapy group and $62 \%$ of the chemoradiation group actually started their postoperative treatment. Our current study also demonstrated a low completion rate of adjuvant chemotherapy after neoadjuvant chemotherapy. Unfortunately, limited granularity of details in the NCDB makes it difficult to determine causality of the low completion rates. Potential reasons may be deconditioning after surgery, post-operative complications, or subjective patient preference. Given the poor completion rates for postoperative therapy, interest has shifted towards optimizing preoperative regimens.

It is notable that the two evidence-based regimens supported by the INT-0116 and MAGIC trials only comprised 37.7\% of all the patients in the study cohort in 2015. A large portion of the remainder of patients underwent neoadjuvant 
Table 4 Comparison of patient and tumor factors between patients receiving perioperative chemotherapy vs. adjuvant chemoradiation in the sub-analysis of patients diagnosed in 2010-2015 with clinical stage III disease

\begin{tabular}{|c|c|c|c|}
\hline & $\begin{array}{l}\text { Perioperative } \\
\text { chemotherapy } \\
(n=320) N(\%) \\
\text { or median } \\
\text { (IQR) }\end{array}$ & $\begin{array}{l}\text { Adjuvant } \\
\text { chemoradiation } \\
(n=176) N(\%) \\
\text { or median } \\
(\mathrm{IQR})\end{array}$ & $\begin{array}{l}p \\
\text { value }\end{array}$ \\
\hline Age & $62(54-70)$ & $64(55-74)$ & 0.04 \\
\hline Male gender & $231(72.2 \%)$ & $122(69.3 \%)$ & \\
\hline Ethnicity & & & 0.01 \\
\hline Caucasian & $232(73.4 \%)$ & $107(61.1 \%)$ & \\
\hline Black & $46(14.6 \%)$ & $48(27.4 \%)$ & \\
\hline Asian & $29(9.2 \%)$ & $17(9.7 \%)$ & \\
\hline American Indian & $1(0.3 \%)$ & $0(0 \%)$ & \\
\hline Other/unknown & $8(2.5 \%)$ & $3(1.7 \%)$ & \\
\hline Insurance & & & 0.43 \\
\hline Private & $142(44.4 \%)$ & $74(42.8 \%)$ & \\
\hline Medicaid & $33(10.3 \%)$ & $13(7.5 \%)$ & \\
\hline Medicare & $132(41.3 \%)$ & $73(42.2 \%)$ & \\
\hline Not insured & $10(3.1 \%)$ & $11(6.4 \%)$ & \\
\hline Unknown & $3(0.9 \%)$ & $2(1.2 \%)$ & \\
\hline Income & & & 0.60 \\
\hline$<\$ 30,000$ & $41(13.6 \%)$ & $31(18.2 \%)$ & \\
\hline$\$ 30,000-34,999$ & $51(16.9 \%)$ & $28(16.5 \%)$ & \\
\hline$\$ 35,000-\$ 45,999$ & $86(28.5 \%)$ & $45(26.5 \%)$ & \\
\hline$\$ 46,000+$ & $124(41.1 \%)$ & $66(38.8 \%)$ & \\
\hline Facility type & & & $<0.01$ \\
\hline $\begin{array}{l}\text { Community cancer } \\
\text { programs }\end{array}$ & $12(4.2 \%)$ & $20(13.3 \%)$ & \\
\hline $\begin{array}{l}\text { Academic/research pro- } \\
\text { gram }\end{array}$ & $194(68.1 \%)$ & $56(37.3 \%)$ & \\
\hline $\begin{array}{l}\text { Integrated Network } \\
\text { Cancer Program }\end{array}$ & $79(27.7 \%)$ & $74(49.3 \%)$ & \\
\hline Location & & & 0.01 \\
\hline Urban & $310(99.7 \%)$ & $163(96.5 \%)$ & \\
\hline Rural & $1(0.3 \%)$ & $6(3.6 \%)$ & \\
\hline Charlson-Deyo Score & & & 0.19 \\
\hline 0 & $231(72.2 \%)$ & $125(71.0 \%)$ & \\
\hline 1 & $75(23.4 \%)$ & $36(20.5 \%)$ & \\
\hline 2 & $8(2.5 \%)$ & $11(6.3 \%)$ & \\
\hline 3 & $6(1.9 \%)$ & $4(2.3 \%)$ & \\
\hline Path stage & & & $<0.01$ \\
\hline 0 & $5(1.8 \%)$ & $0(0 \%)$ & \\
\hline $\mathrm{I}$ & $32(11.6 \%)$ & $1(0.6 \%)$ & \\
\hline II & $89(32.4 \%)$ & $10(6.1 \%)$ & \\
\hline III & $140(50.9 \%)$ & $152(93.3 \%)$ & \\
\hline IV & $9(3.3 \%)$ & $0(0 \%)$ & \\
\hline Tumor site & & & $<0.01$ \\
\hline Cardia/fundus & $155(48.4 \%)$ & $26(14.8 \%)$ & \\
\hline Body/antrum & $82(25.6 \%)$ & $67(38.1 \%)$ & \\
\hline Other/unknown & $83(25.9 \%)$ & $83(47.2 \%)$ & \\
\hline
\end{tabular}

Table 4 (continued)

\begin{tabular}{|c|c|c|c|}
\hline & $\begin{array}{l}\text { Perioperative } \\
\text { chemotherapy } \\
(n=320) N(\%) \\
\text { or median } \\
\text { (IQR) }\end{array}$ & $\begin{array}{l}\text { Adjuvant } \\
\text { chemoradiation } \\
(n=176) N(\%) \\
\text { or median } \\
\text { (IQR) }\end{array}$ & $\begin{array}{l}p \\
\text { value }\end{array}$ \\
\hline Grade & & & 0.22 \\
\hline Well-differentiated & $10(3.5 \%)$ & $1(0.6 \%)$ & \\
\hline Moderately differentiated & $85(29.5 \%)$ & $50(29.8 \%)$ & \\
\hline Poorly differentiated & $189(65.5 \%)$ & $116(69.1 \%)$ & \\
\hline Unknown & $4(1.4 \%)$ & $1(0.6 \%)$ & \\
\hline Type of resection & & & $<0.01$ \\
\hline $\begin{array}{l}\text { Total/near total } \\
\text { gastrectomy }\end{array}$ & $131(43.4 \%)$ & $47(27.7 \%)$ & \\
\hline Partial gastrectomy & $171(56.6 \%)$ & $123(72.4 \%)$ & \\
\hline Lymph nodes examined & & & $<0.01$ \\
\hline 0 & $13(4.1 \%)$ & $3(1.7 \%)$ & \\
\hline $1-14$ & $47(14.7 \%)$ & $49(27.8 \%)$ & \\
\hline $15+$ & $258(80.6 \%)$ & $122(69.3 \%)$ & \\
\hline Unknown & $2(0.6 \%)$ & $2(1.1 \%)$ & \\
\hline Positive lymph nodes & $194(63.2 \%)$ & $160(92.5 \%)$ & $<0.01$ \\
\hline Margin status & & & 0.03 \\
\hline $\mathrm{R} 0$ & $286(94.4 \%)$ & $130(87.3 \%)$ & \\
\hline $\mathrm{R} 1$ & $15(5.0 \%)$ & $16(10.7 \%)$ & \\
\hline $\mathrm{R} 2$ & $2(0.7 \%)$ & $3(2.0 \%)$ & \\
\hline
\end{tabular}

chemoradiation. While the benefit of neoadjuvant chemoradiation has been generally accepted for gastroesophageal junction tumors, ${ }^{18,19}$ its role in the management of resectable gastric cancer remains limited to retrospective studies and a small phase II study that included gastric and GEJ tumors. ${ }^{20-23} \mathrm{In}$ this current study, it is important to note that the majority of tumors treated with this approach were located in the cardia or fundus. While we tried to eliminate as many GEJ tumors as possible by excluding patients who also underwent partial esophagectomy with their cancer-targeted surgical intervention, it is possible that some GEJ tumors may still be included in the cohort if they did not undergo esophageal resection. It is likely that treatment of the cardia/fundus tumors was extrapolated from standard treatment of GEJ tumors. A previous analysis of the NCDB found that neoadjuvant chemoradiation was associated with a higher pathologic complete response rate and $\mathrm{R} 0$ resection, but was not associated with improved overall survival compared with neoadjuvant chemotherapy in gastric cancer. ${ }^{24} \mathrm{~A}$ retrospective study at MD Anderson Cancer Center analyzing outcomes after neoadjuvant chemoradiation and surgery suggested that signet ring cell histology may be associated with higher resistance to neoadjuvant chemoradiation. ${ }^{25}$ This suggests that treatment strategies may be optimized by individualized selection based on tumor biology. The ongoing CRITICS-II trial comparing 
Table 5 Multivariate Cox regression analysis for predictors of overall survival in patients diagnosed between 2010 and 2015 with clinical stage III disease

\begin{tabular}{l}
\hline \\
\hline Ethnicity \\
Caucasian \\
Black \\
Asian \\
American Indian \\
Other/unknown \\
Insurance \\
Private \\
Medicaid \\
Medicare \\
Not insured \\
Unknown \\
Income \\
$<\$ 30,000$ \\
$\$ 30,000-34,999$ \\
$\$ 35,000-\$ 45,999$ \\
$\$ 46,000+$ \\
Facility type \\
Academic/research program \\
Community cancer programs \\
Integrated Network Cancer Program \\
Location \\
Rural \\
Urban \\
Charson-Deyo Com
\end{tabular}

Charlson-Deyo Comorbidity Score

0

1

2

3

Treatment regimen

Surgery alone

Perioperative chemotherapy

Adjuvant chemoradiation

Neoadj chemoradiation

Other (adjuvant chemotherapy or adjuvant radiation)

Pathologic stage

0

I

II

III

IV

Tumor site

Body/antrum

Cardia/fundus

Other/unknown

Grade

$0.75(0.52-1.10) \quad 0.14$

$0.87(0.61-1.24) \quad 0.45$

$0.86(0.62-1.21) \quad 0.39$

Ref.

$1.06(0.74-1.51) \quad 0.77$

$1.15(0.92-1.44) \quad 0.22$

Ref.

$0.60(0.31-1.18)$

Ref.

$1.09(0.86-1.39)$

0.48

$1.30(0.83-2.05) \quad 0.26$

$1.14(0.52-2.50) \quad 0.74$

Ref.

$0.49(0.35-0.68)<0.01$

$0.31(0.21-0.44)<0.01$

$0.55(0.40-0.76)<0.01$

$0.41(0.27-0.64)<0.01$

$0.82(0.27-2.52) \quad 0.73$

Ref.

$1.51(0.84-2.71) \quad 0.17$

$3.65(2.00-6.66)<0.01$

$7.47(3.57-15.65) \quad<0.01$

Ref.

$1.29(0.92-1.81) \quad 0.15$

$1.23(0.89-1.70) \quad 0.22$
Table 5 (continued)

\begin{tabular}{llc}
\hline & HR $(95 \%$ CI $)$ & $p$ value \\
\hline Well-differentiated & Ref. & \\
Moderately differentiated & $2.00(1.02-3.91)$ & 0.04 \\
Poorly differentiated & $2.24(1.17-4.29)$ & 0.02 \\
Unknown & $2.54(0.93-6.95)$ & 0.07 \\
Type of resection & & \\
Total/near total gastrectomy & Ref. & \\
Partial gastrectomy & $0.94(0.75-1.17)$ & 0.56 \\
Lymph nodes examined & & \\
1-14 & Ref. & \\
15+ & $0.80(0.64-1.00)$ & 0.049 \\
Positive lymph nodes & $0.97(0.70-1.35)$ & 0.85 \\
Margin status & & \\
R0 & Ref. & $<0.01$ \\
R1 & $2.55(1.82-3.56)$ & 0.59 \\
R2 & $0.77(0.30-2.00)$ & \\
\hline
\end{tabular}

neoadjuvant chemotherapy versus neoadjuvant chemotherapy plus chemoradiation versus neoadjuvant chemoradiation will hopefully provide more evidence regarding the optimal preoperative treatment strategy for resectable gastric cancer. ${ }^{26}$ Likewise, the ongoing TOPGEAR trial comparing perioperative chemotherapy with versus without neoadjuvant chemoradiation may shed light on potential benefit of preoperative chemoradiation in addition to chemotherapy. ${ }^{27}$

There are limitations to this study which must be addressed. The retrospective nature of the study limits

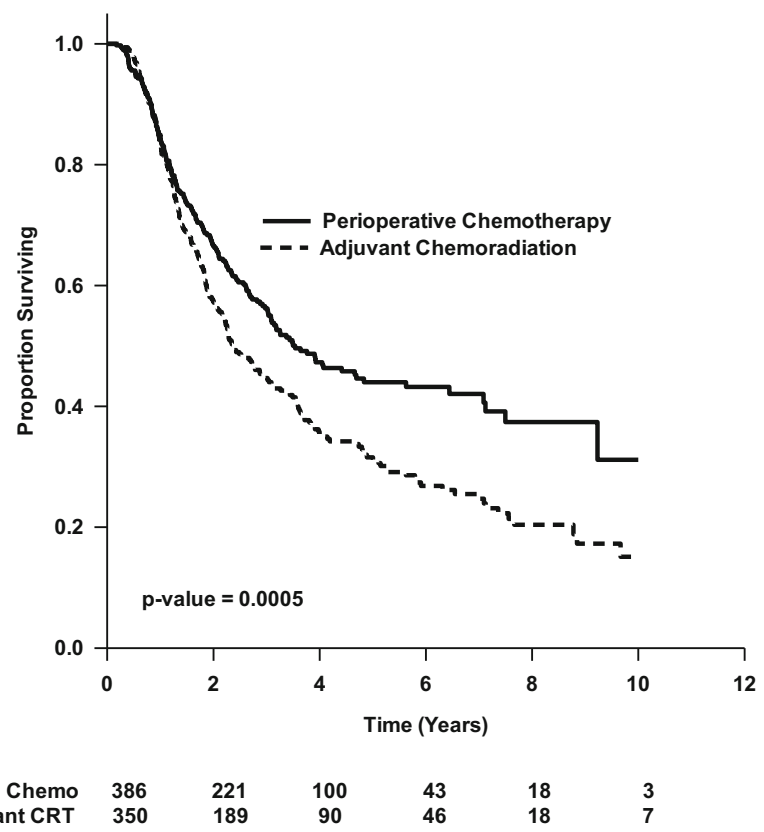

Fig. 5 Improved overall survival in clinical stage III gastric adenocarcinoma for perioperative chemotherapy when compared with adjuvant chemoradiation 


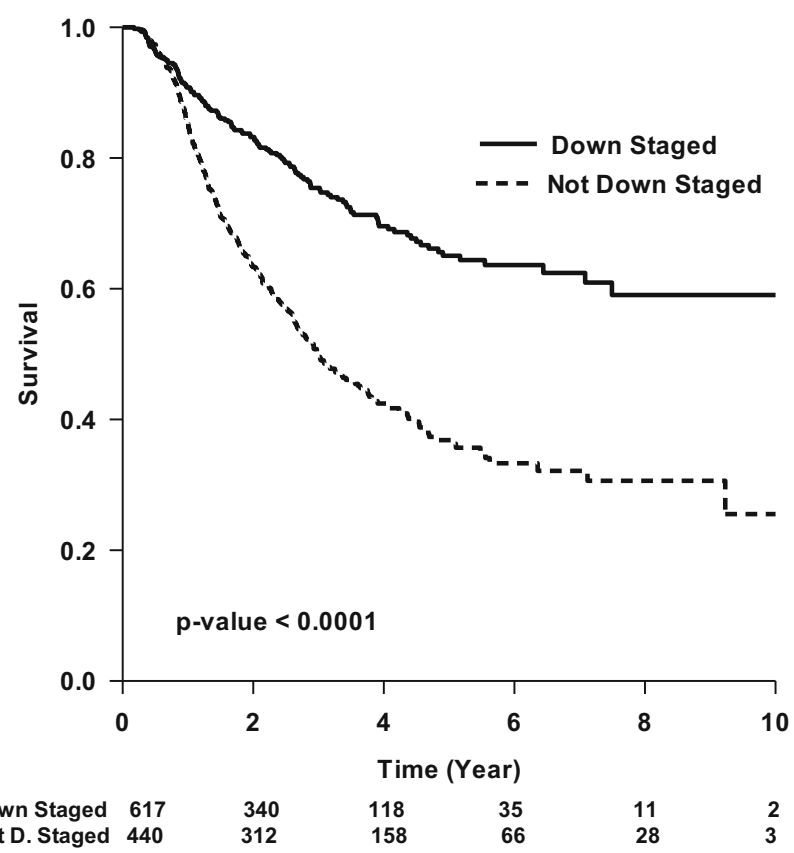

Fig. 6 Kaplan-Meier analysis comparing overall survival of patients who underwent neoadjuvant chemotherapy and were either down-staged (between their clinical and pathologic stages) or were not down-staged

conclusions to showing associations, without the ability to demonstrate causality. There may also be inherent selection bias, which is difficult to account for in retrospective studies. However, the data in the NCDB portrays real-world practice patterns across the USA, which is important to be aware of when investigating potential time lags in the adoption of level 1 evidence. In our analyses, we chose to use neoadjuvant chemotherapy as a representative surrogate for perioperative chemotherapy, with the intention to treat assumption that if patients were given neoadjuvant chemotherapy, it was planned that they would also receive adjuvant chemotherapy. However, there was a low rate of receipt of adjuvant chemotherapy, and due to limitations in the details of the database, we are unable to determine the reasons behind the low compliance rate. These low completion rates for postoperative treatment are not new to gastric cancer patients as similarly low rates were seen in the MAGIC, CRITICS, and FLOT4 trials. However, even with low completion of adjuvant chemotherapy, the perioperative chemotherapy was still associated with better survival compared with adjuvant radiation or other regimens. Another limitation is the possibility of inclusion of gastroesophageal junction tumors in the analysis. In order to address this, we excluded patients who also underwent esophageal resection as part of their cancerdirected surgical intervention as these were most likely patients with gastroesophageal junction tumors. Despite this, a proportion of these patients may still be included in the analysis and may represent patients that underwent proximal gastrectomy plus esophagectomy with the latter not being captured in the dataset. Additionally, one other point should be made regarding selection bias and the lack of granularity of a national database. It is unfortunately impossible to identify patients with underlying significant morbidity, deconditioning, or decreased performance status that may have precluded meaningful neoadjuvant therapy and that upfront resection may not only be the most reasonable treatment but also of substantial benefit compared with a palliative approach.

\section{Conclusions}

Since publication of the MAGIC trial, utilization of evidence-based treatments for resectable gastric adenocarcinoma has increased, with perioperative chemotherapy surpassing adjuvant CRT as the preferred practice. However, overall utilization of these regimens remains quite low despite association with improved survival. Further investigation is needed to understand reasons behind continued use of non-evidence-based treatment regimens.

Authors' Contributions All persons who meet authorship criteria are listed as authors, and all authors certify that they have participated sufficiently in the work to take public responsibility for the content, including participation in the concept, design, analysis, writing, or revision of the manuscript. Furthermore, each author certifies that this material or similar material has not been and will not be submitted to or published in any other publication before its appearance in the Journal of Gastrointestinal Surgery.

Category 1

Conception and design of study: TCL, SHP, GCW

Acquisition of data: TCL, KW

Analysis and/or interpretation of data: TCL, KW, MCM, MEJ, SAS, SAA, SHP, GCW

Category 2

Drafting the manuscript: TCL, GCW

Revising the manuscript critically for important intellectual content: TCL, KW, MCM, MEJ, SAS, SAA, SHP, GCW

Category 3

Final approval of the version of the manuscript to be published: TCL, KW, MCM, MEJ, SAS, SAA, SHP, GCW

Category 4

Agreement to be accountable for all aspects of the work: TCL, KW, MCM, MEJ, SAS, SAA, SHP, GCW

Funding University of Cincinnati Department of Surgery.

\section{Compliance with ethical standards}

Conflict of Interest The authors declare that they have no conflict of interest.

\section{References}

1. Ahmad SA, Xia BT, Bailey CE, et al. An update on gastric cancer. Curr Probl Surg. 2016;53(10):449-490. 
2. Macdonald JS, Smalley SR, Benedetti J, et al. Chemoradiotherapy after surgery compared with surgery alone for adenocarcinoma of the stomach or gastroesophageal junction. $N$ Engl J Med. 2001;345(10):725-730.

3. Cunningham D, Allum WH, Stenning SP, et al. Perioperative chemotherapy versus surgery alone for resectable gastroesophageal cancer. N Engl J Med. 2006;355(1):11-20.

4. Gastric Cancer. National Comprehensive Cancer Network. NCCN Clinical Practice Guidelines in Oncology Web site. https://www. nccn.org/professionals/physician_gls/PDF/gastric.pdf. Published 2019. Updated December 20, 2019. Accessed.

5. Snyder RA, Penson DF, Ni S, Koyama T, Merchant NB. Trends in the use of evidence-based therapy for resectable gastric cancer. $J$ Surg Oncol. 2014;110(3):285-290.

6. Sherman KL, Merkow RP, Bilimoria KY, et al. Treatment trends and predictors of adjuvant and neoadjuvant therapy for gastric adenocarcinoma in the United States. Ann Surg Oncol. 2013;20(2): 362-370.

7. Mokdad AA, Ali A, Yopp AC, et al. Adoption of evidence-based novel therapies in the treatment of gastric cancer: a national observational study. Cancer. 2018;124(6):1122-1131.

8. National Cancer Database. American College of Surgeons. https:// www.facs.org/quality-programs/cancer/ncdb. Published 2020. Accessed.

9. Al-Batran SE, Homann N, Pauligk C, et al. Perioperative chemotherapy with fluorouracil plus leucovorin, oxaliplatin, and docetaxel versus fluorouracil or capecitabine plus cisplatin and epirubicin for locally advanced, resectable gastric or gastro-oesophageal junction adenocarcinoma (FLOT4): a randomised, phase 2/3 trial. Lancet. 2019;393(10184):1948-1957.

10. Morris ZS, Wooding S, Grant J. The answer is 17 years, what is the question: understanding time lags in translational research. $J R$ Soc Med. 2011;104(12):510-520.

11. Cohen DJ, Leichman L. Controversies in the treatment of local and locally advanced gastric and esophageal cancers. J Clin Oncol. 2015;33(16):1754-1759

12. Fitzgerald TL, Efird JT, Bellamy N, et al. Perioperative chemotherapy versus postoperative chemoradiotherapy in patients with resectable gastric/gastroesophageal junction adenocarcinomas: a survival analysis of 5058 patients. Cancer. 2017;123(15):2909-2917.

13. Stumpf PK, Amini A, Jones BL, et al. Adjuvant radiotherapy improves overall survival in patients with resected gastric adenocarcinoma: a National Cancer Data Base analysis. Cancer. 2017;123(17):3402-3409.

14. Jabo B, Selleck MJ, Morgan JW, et al. Comparison of perioperative chemotherapy with adjuvant chemoradiotherapy for resectable gastric cancer: findings from a population-based study. $J$ Gastrointest Oncol. 2018;9(1):35-45.

15. Park SH, Sohn TS, Lee J, et al. Phase III trial to compare adjuvant chemotherapy with capecitabine and cisplatin versus concurrent chemoradiotherapy in gastric cancer: final report of the adjuvant chemoradiotherapy in stomach tumors trial, including survival and subset analyses. J Clin Oncol. 2015;33(28):3130-3136.

16. Park SH, Zang DY, Han B, et al. ARTIST 2: Interim results of a phase III trial involving adjuvant chemotherapy and/or chemoradiotherapy after D2-gastrectomy in stage II/III gastric cancer (GC). Journal of Clinical Oncology. 2019;37(15_suppl):40014001.

17. Cats A, Jansen EPM, van Grieken NCT, et al. Chemotherapy versus chemoradiotherapy after surgery and preoperative chemotherapy for resectable gastric cancer (CRITICS): an international, open-label, randomised phase 3 trial. Lancet Oncol. 2018;19(5):616-628.

18. Meng X, Wang L, Zhao Y, et al. Neoadjuvant chemoradiation treatment for resectable esophago-gastric cancer: a systematic review and meta-analysis. $J$ Cancer. 2019;10(1):192-204.

19. Zhao X, Ren Y, Hu Y, Cui N, Wang X, Cui Y. Neoadjuvant chemotherapy versus neoadjuvant chemoradiotherapy for cancer of the esophagus or the gastroesophageal junction: a meta-analysis based on clinical trials. PLoS One. 2018;13(8):e202185.

20. Lowy AM, Feig BW, Janjan N, et al. A pilot study of preoperative chemoradiotherapy for resectable gastric cancer. Ann Surg Oncol. 2001;8(6):519-524.

21. Ajani JA, Mansfield PF, Janjan N, et al. Multi-institutional trial of preoperative chemoradiotherapy in patients with potentially resectable gastric carcinoma. J Clin Oncol. 2004;22(14):2774-2780.

22. Ajani JA, Mansfield PF, Crane CH, et al. Paclitaxel-based chemoradiotherapy in localized gastric carcinoma: degree of pathologic response and not clinical parameters dictated patient outcome. $J$ Clin Oncol. 2005;23(6):1237-1244.

23. Rivera F, Galan M, Tabernero J, et al. Phase II trial of preoperative irinotecan-cisplatin followed by concurrent irinotecan-cisplatin and radiotherapy for resectable locally advanced gastric and esophagogastric junction adenocarcinoma. Int J Radiat Oncol Biol Phys. 2009;75(5):1430-1436.

24. Ikoma N, Das P, Hofstetter W, et al. Preoperative chemoradiation therapy induces primary-tumor complete response more frequently than chemotherapy alone in gastric cancer: analyses of the National Cancer Database 2006-2014 using propensity score matching. Gastric Cancer. 2018;21(6):1004-1013.

25. Charalampakis N, Nogueras Gonzalez GM, Elimova E, et al. The proportion of signet ring cell component in patients with localized gastric adenocarcinoma correlates with the degree of response to pre-operative chemoradiation. Oncology. 2016;90(5):239-247.

26. Slagter AE, Jansen EPM, van Laarhoven HWM, et al. CRITICS-II: a multicentre randomised phase II trial of neo-adjuvant chemotherapy followed by surgery versus neo-adjuvant chemotherapy and subsequent chemoradiotherapy followed by surgery versus neoadjuvant chemoradiotherapy followed by surgery in resectable gastric cancer. BMC Cancer. 2018;18(1):877.

27. Leong T, Smithers BM, Haustermans K, et al. TOPGEAR: a randomized, phase III trial of perioperative ecf chemotherapy with or without preoperative chemoradiation for resectable gastric cancer: interim results from an international, intergroup trial of the AGITG, TROG, EORTC and CCTGAnn Surg Oncol. 2017;24(8):22522258.

Publisher's Note Springer Nature remains neutral with regard to jurisdictional claims in published maps and institutional affiliations. 